\title{
The elastic scattering of an electron from the target BY absorbing a photon via free- free scattering theory
}

\author{
Kishori Yadav, Jeevan Jyoti Nakarmi* \\ Central Department of Physics, T. U., Kirtipur, Nepal
}

Email address:

nakarmijj@gmail.com (J. J. Nakarmi)

To cite this article:

Kishori Yadav, Jeevan Jyoti Nakarmi. The Elastic Scattering of an Electron from the Target BY Absorbing a Photon via Free- Free Scattering Theory. International Journal of Mechanical Engineering and Applications. Vol. 2, No. 6, 2014, pp. 87-97.

doi: 10.11648/j.ijmea.20140206.12

\begin{abstract}
This paper intended to the elastic scattering of an electron from the target by absorbing a photon from the laser field has been studied for the polarized potential. Since the solution of the Schrödinger equation of whole three-body system has not been found, we consider such intensities of electromagnetic field (Laser field) that the electron field coupling is the dominant process and the target is transparent to the field such that photon- target coupling can be ignored. Therefore the internal structure of target can be ignored and represented just as a scattering potential. For number of photon, $l=-1$ i.e, absorption of a photon (inverse Bremsstrahlung), we have concluded that the differential scattering cross section of an electron depends upon the fourth power of the wavelength $\left(\lambda^{4}\right)$ and the intensity of the Laser field. From this work we see that at certain values of laser parameters the differential scattering cross section of scattered electron decreases with increase in scattering angle and attains a minimum value of 0.1 barn and further increase in scattering angle also increases in differential scattering cross section and attains a maximum value of 0.3 barn.
\end{abstract}

Keywords: Scattering, Bremsstrahlung, Volkov Wave Function, Polarized Potential

\section{Introduction}

Electron atom interaction in the presence of a laser field attracted considerable theoretical attention in the recent year not only because of the importance in applied areas (such as plasma heating or laser driven fusion), but also in view of their interest in fundamental atomic theory. The problem of this process, is in general, very complex, since in addition to the difficulties associated with the treatment of electron atom collision, the presence of the laser introduces new parameters (for example, the laser photon energy $\hbar \omega$ and intensity I) which may influence the collision. Moreover, the laser photon can play the role of a "third body" during the collision, and "dressed" the atomic states. It is therefore of interest to begin the theoretical analysis by considering the simpler problem of the scattering of an electron by a potential in the presence of a laser field. A fully realistic description of the target atom is quite difficult. We shall represent it here by a potential model.

Mason and Newell (1982) reported experimental evidence of simultaneous electron-photon excitation of atoms. However most experimental studies have been performed with noble gases (Wallbank et al. 2009), a recent on being with a Nd:YAG laser (Luan et al 2011). On the other hand, theoretical studies are not easy to perform with these atoms and the hydrogen atom has been studied extensively (Rahman ans Faisal 1978, Jetzek et al 1988, Bhattacharya et al 1993). Hydrogen as a one electron atom is a simple to deal with and it is often interesting information concerning the main features of the problem.

The free process can theoretically be studied at various levels. As the target does not change states in this process, its own energy spectrum can be ignored and a simple potential can mimic the electron atom interaction. The collision process can then be treated either classically or quantum mechanically by means of the simple scattering theories . Furthermore, the collision can be treated as occurred at such intensities of electromagnetic field that the electron-field coupling is the dominant process and the target is transparent to the field such that photon-target coupling can be ignored. If, however, the frequency of the photon is such as to couple two stationary states of the target, then the target-field interaction becomes extremely important. Here we discussed such intensity of the electromagnetic field where the photon- 
field interaction can be neglected.

We want to show the effect of various collision and laser parameters on the collision process. This is the motive of the paper. Furthermore, most theoretical studies for scattering of electron by atoms in an intense radiation field are based on perturbation theory (Gersten and Mittleman 1976; Byron and Joachain 1984; Garvila et al 1990) starting with the well known Kroll and Waston (1976) work on the soft photon approximation, there exist only a few non-perturbative approaches for this problem. Shakeshaft (1983) formulated a non- perturbative method of coupled integral equations for calculating the scattering cross section by assuming the potential to be separable. Rosenberg (2000) applied the variation method for coulomb scattering in a laser field using a low frequency approximation.

The reaction studied in the present work is,

$$
e_{k_{i}}^{-}+H(i)+N(\omega, \hat{\epsilon}) \rightarrow e_{k_{f}}^{-}+H(j)+(N \pm l) \gamma(\omega, \hat{\epsilon})
$$

Representing the collision of an incoming electron with momentum $k_{i}$ with hydrogen atom initially in the state $\mathrm{i}$ in the presence of a single mode laser beam moving to the excited state $\mathrm{j}$ with exchange of 1 photons between the electron and the laser field. We have used the Born approximation, to treat the electron- atom interaction as it is simple enough to allow calculation of a larger number of transitions, and it becomes exact at high energies.

\section{Materials and Methods}

We consider a collision between an electron and hydrogen atom in the presence of a laser field. We begin this section by considering the simple case in which the target atom is modeled by a center of force and hence does not interact with the laser field. The field is assumed to be purely monochromatic with angular frequency $\omega$, linearly polarized with linear polarization vector $\hat{\epsilon}$. We also assume that the dipole approximation is valid. The Hamiltonian of the electron-atom system in the presence of a laser beam can be written as,

$$
H=H_{f}+H_{t}+V\left(r_{o}, r_{a}\right)
$$

Where

$H_{f} \rightarrow$ Hamiltonian of the free electron

$H_{t} \rightarrow$ Hamiltonian of the target atom

And $V\left(r_{o}, r_{a}\right)$ is the interaction between the incident electron and atom, defined as,

$$
V\left(r_{o}, r_{a}\right)=\frac{-Z}{\left|r_{o}\right|}+\sum_{j=1}^{Z} \frac{1}{\left|r_{o j}\right|}
$$

Where,

$r_{o} \rightarrow$ Position coordinate of the projectile electron

$\mathrm{Z} \rightarrow$ Atomic number of target or charge of the target atom

$r_{a} \rightarrow$ Position of an atomic electron

For H-atom, $\mathrm{Z}=1$ (i.e, one electron) equation (2) becomes

$$
V\left(r_{o}, r_{a}\right)=\frac{-Z}{\left|r_{o}\right|}+\frac{1}{\left|r_{o}-r_{1}\right|}
$$

In equation (2) $\mathrm{Z}$ is the charge of the target atom. Working in the coulomb gauge we have the electric field

$$
\vec{E}(t)=\vec{E}_{o} \sin \omega t
$$

And the corresponding vector potential is

$$
\vec{A}(t)=\vec{A}_{o} \cos \omega t \text { with } \vec{A}_{o}=\frac{c \vec{E}_{o}}{\omega}
$$

In the presence of a laser field the incidence electron of momentum $k$ is represented as

$$
\chi\left(\vec{r}_{o}, t\right)=(2 \pi)^{\frac{-3}{2}} \exp \left[\left(i \vec{k} \cdot \vec{r}-i \vec{k} \cdot \vec{\alpha}_{o} \sin \omega t\right)-\frac{i E_{k} t}{\hbar}\right]
$$

Where,

$$
\begin{aligned}
& E_{k}=\frac{\hbar^{2} k^{2}}{2 m} \\
& \vec{\alpha}_{o}=\frac{e \vec{A}_{o}}{m c \omega}
\end{aligned}
$$

Also, $\vec{\alpha}_{o}=\frac{e \vec{E}_{o}}{m \omega^{2}}$ is the measure of the coupling between the field and the projectile.

Equation (3) is also known as volkov wave function. This equation gives the states of a free electron in a laser field. Since there is no conservation of energy in free state of an electron. So the states repersented by the volkov wave functiion is also called as virtual state, to make the energy conservation there must be another parameter. Here in our case we take the static potential as the third parameter for energy conservation of electron.

The S-matrix (scattering matrix) element for the transition from initial state (i) to final state (f) $(i . e i \rightarrow f)$ is given by, [ref]

$$
S=\frac{-i}{\hbar}<\chi_{f} V \psi_{i}^{+}>
$$

The angle bracket denotes both space and time integration. Where,

$\chi_{f}$ is the volkov wave function for the final state of an electron

$\psi_{i}^{+}$is the exact formal solution of the following schrödinger wave equation,

$$
\frac{1}{2 m}\left(\vec{P}+\frac{e \vec{A}(t)}{c}\right)^{2} \psi(\vec{r}, t)+V(\vec{r}) \psi(\vec{r}, t)=i \hbar \frac{\partial \psi(\vec{r}, t)}{\partial t}
$$

where $\psi(\vec{r}, t)$ is the time dependent wave function in presence of the scattering potential $V(\vec{r})$ in the presence of laser field.

the solution this equation (4.65) can be written as [ref],

$$
\psi^{+}(\vec{r}, t)=\psi_{i}(\vec{r}, t)+\int_{-\infty}^{\infty} G_{+}\left(t, t^{\prime}\right) V \psi^{+}\left(\vec{r}, t^{\prime}\right) d t^{\prime}
$$

Now taking electron atom interaction upto the first Born approximation, we get scattering matrix (S-matrix) as,

$$
S=\frac{-i}{\hbar}<\chi_{f}|V| \chi_{i}>
$$


$\chi_{i}$ is the Volkov wave function for the initial state of an electron

And $\mathrm{V}$ is the interaction potential defined by equation (2).

Initially the electron is free from potential and take part in interaction for certain time and after that it scattered from the potential to the Free State. So this interaction is also known as free- free scattering process.

Now we can write equation (4) as,

$$
\begin{aligned}
S_{k_{f} k_{i}}=\frac{-i}{\hbar} \iint_{-\infty}^{t} & (2 \pi)^{\frac{-3}{2}} \exp \left[\left(i \vec{k}_{f} \cdot \vec{r}-i \vec{k}_{f} \cdot \vec{\alpha}_{o} \sin \omega t\right)\right. \\
& \left.-\frac{i E_{k_{f}} t}{\hbar}\right] V(\vec{r})(2 \pi)^{\frac{-3}{2}} \exp \left[\left(i \vec{k}_{i} \cdot \vec{r}\right.\right. \\
& \left.\left.-i \vec{k}_{i} \cdot \vec{\alpha}_{o} \sin \omega t\right)-\frac{i E_{i} t}{\hbar}\right] d^{3} r d t
\end{aligned}
$$

Where,

$k_{i} \rightarrow$ Initial wave vector of projectile particle

$k_{f} \rightarrow$ Final wave vector of the scattered particle

$=\frac{-i}{\hbar}(2 \pi)^{-3} \iint_{-\infty}^{t} e^{-i \Delta \cdot \vec{r}} e^{i \Delta \cdot \vec{\alpha}_{o} \sin \omega t} e^{i\left(E_{k_{f}}-E_{k_{i}}\right) \frac{t}{\hbar}} V(\vec{r}) d^{3} r d t$

Here, $\Delta \rightarrow\left(\vec{k}_{f}-\vec{k}_{i}\right)$

(momentum transfer during scattering process)

$$
S_{k_{f} k_{i}}=\frac{-i}{\hbar} \widehat{V}(\Delta) \int_{-\infty}^{t} e^{i \Delta \cdot \vec{\alpha}_{o} \sin \omega t} e^{i\left(E_{k_{f}}-E_{k_{i}}\right) \frac{t}{\hbar}} d t
$$

where,

$$
\widehat{V}(\Delta)=(2 \pi)^{-3} \int e^{-i \Delta \cdot \vec{r}} V(\vec{r}) d^{3} r
$$

This is the Fourier transformation of the potential in the momentum space.

Since $V(\vec{r})$ is independent of time $(\mathrm{t})$, we can seperate time and space integration so that $\hat{V}(\Delta)$ can be taken outside of time integration.

Using the generating function of the Bessel Polynomial

We get,

$$
e^{i \Delta \cdot \alpha_{o} \sin \omega t}=\sum_{l=-\infty}^{\infty} J_{l}\left(\Delta . \alpha_{o}\right) e^{i l \omega t}
$$

So the S-matrix element becomes

$$
S_{k_{f} k_{i}}=\frac{-i}{\hbar} \hat{V}(\Delta) \int_{-\infty}^{t} \sum_{l=-\infty}^{\infty} J_{l}\left(\Delta . \alpha_{o}\right) e^{i l \omega t} e^{i\left(E_{k_{f}}-E_{k_{i}}\right) \frac{t}{\hbar}} d t
$$

where $l$ is the no of photons exchange during the scattering process. It may take the values,

$$
l=0, \pm 1, \pm 2, \pm 3, \ldots \ldots \ldots \ldots
$$

Where, positive $l$ describes the photon emission stimulated
Bremsstrahlung and negative $l$ describes the photon absorption or inverse Bremsstrahlung and $l=0$ corresponds to the pure elastic scattering in the presence of the laser field.

$$
S_{k_{f} k_{i}}=\frac{-i}{\hbar} T_{k_{f} k_{i}}^{l} \int_{-\infty}^{t} e^{i\left(E_{k_{f}}-E_{k_{i}}+l \hbar \omega\right) \frac{t}{\hbar}} d t
$$

where,

$$
T_{k_{f} k_{i}}^{l}=\hat{V}(\Delta) \sum_{l} J_{l}\left(\Delta . \alpha_{o}\right)
$$

Using the idea of integral form of Dirac delta function

$$
\left(\delta\left(x-x^{\prime}\right)=\frac{1}{2 \pi} \int_{-\infty}^{\infty} e^{i k\left(x-x^{\prime}\right)} d k\right)
$$

We reduce equation (5) in the following form,

$$
S_{k_{f} k_{i}}=-2 \pi i T_{k_{f} k_{i}}^{l} \delta\left(E_{k_{f}}-E_{k_{i}}+l \hbar \omega\right)
$$

The delta function in above equation ensures energy conservation,

$$
\text { i.e, } \begin{aligned}
& E_{k_{f}}=E_{k_{i}}-l \hbar \omega \\
& \frac{k_{f}}{k_{i}}=\left(1-\frac{l \hbar \omega}{E_{k_{i}}}\right)^{\frac{1}{2}}
\end{aligned}
$$

Here $\hbar \omega$ is the photon energy and $E_{k_{i}}$ is the kinetic energy of a projectile electron.

Now the differential scattering cross section in terms of transiton matrix is given by,

$$
\frac{d \sigma^{B 1}}{d \Omega}=\frac{m^{2}}{(2 \pi)^{2} \hbar^{4}} \frac{k_{f}}{k_{i}}\left|T_{k_{f} k_{i}}^{l}\right|^{2}
$$

This is the required relation of differential cross section of electron with transfer of $l$ photon.

where,

$$
\begin{aligned}
& \left|T_{k_{f} k_{i}}^{l}\right|^{2}=\sum_{l} J_{l}^{2}\left(\Delta \cdot \alpha_{o}\right)|\widehat{V}(\Delta)|^{2} \\
& \hat{V}(\Delta)=(2 \pi)^{-3} \int e^{-i \Delta \cdot \vec{r}} V(\vec{r}) d^{3} r
\end{aligned}
$$

For spherically symmetric potential,

$$
V(\vec{r})=V(r)
$$

So that,

$\hat{V}(\Delta)=(2 \pi)^{-3} \int_{0}^{\infty} V(r) r^{2} d r \int_{0}^{\pi} e^{-i \Delta \mathrm{r} \cos \theta} \sin \theta d \theta \int_{0}^{2 \pi} d \phi$

This gives,

$$
|\widehat{V}(\Delta)|^{2}=\frac{e^{4}}{4 \pi^{4} \Delta^{4}}
$$

To calculate $\sum_{l} J_{l}\left(\Delta . \alpha_{o}\right)$ we have the expression for the Bessel function which is, [ref] 
$J_{n}(x)=\frac{x^{n}}{2^{n} \Gamma(\mathrm{n}+1)}\left[1-\frac{x^{2}}{2.2(n+1)}+\frac{x^{4}}{2.4 .2(n+1)(n+2)}-\ldots ..\right]$

Here we take

$$
\begin{gathered}
n=l \text { and } x=\Delta . \alpha_{o} \text { then } \\
J_{l}(x)=\frac{\left(\Delta \cdot \alpha_{o}\right)^{l}}{2^{l} \Gamma(l+1)}\left[1-\frac{\left(\Delta \cdot \alpha_{o}\right)^{2}}{2.2(l+1)}+\frac{\left(\Delta \cdot \alpha_{o}\right)^{4}}{2.4 .2(l+1)(l+2)}-\ldots \ldots\right]
\end{gathered}
$$

For low intensity and low frequency we get,

$$
\left[J_{l}\left(\Delta . \alpha_{o}\right)\right]^{2}=\frac{\Delta^{2} \alpha_{o}{ }^{2}}{4}
$$

Thus we get square modulus of the transition matrix element as,

$$
\left|T_{k_{f} k_{i}}^{l}\right|^{2}=\frac{\Delta^{2} \alpha_{o}^{2}}{4} \frac{e^{4}}{4 \pi^{4} \Delta^{4}}
$$

Substituting these values we get the value of the differential cross section as,

$$
\begin{gathered}
\frac{d \sigma^{B 1}}{d \Omega}=\frac{e^{6}}{(2 \pi)^{6} \hbar^{4}} \frac{k_{f}}{k_{i}} \frac{E_{o}{ }^{2}}{\omega^{4}\left(k_{f}-k_{i}\right)^{2}} \\
\frac{d \sigma^{B 1}}{d \Omega}=\frac{e^{6}}{(2 \pi)^{6} \hbar^{4}} \frac{k_{f}}{k_{i}} \frac{E_{o}{ }^{2}}{\omega^{4}(\Delta)^{2}}
\end{gathered}
$$

We have,

$$
\begin{gathered}
\Delta=\overrightarrow{k_{f}}-\overrightarrow{k_{l}} \\
\Delta^{2}=k_{f}^{2}+k_{i}^{2}-2 \overrightarrow{k_{f} k_{\iota}} \cos \theta
\end{gathered}
$$

Where, $\theta$ is the angle between $k_{f}$ and $k_{i}$, that means $\theta$ is the scattering angle between initial and final momentum of the projectile particle.

Thus we have,

$$
\Delta^{2}=k_{i}^{2}\left[\left(1-\frac{l \hbar \omega}{E_{k_{i}}}\right)-2\left(1-\frac{l \hbar \omega}{E_{k_{i}}}\right)^{\frac{1}{2}} \cos \theta+1\right]
$$

Substituting this value in equation (7) we get,

$$
\frac{d \sigma^{B 1}}{d \Omega}=\frac{e^{6}}{(2 \pi)^{6} \hbar^{4}} \frac{k_{f}}{k_{i}} \frac{E_{o}{ }^{2}}{\omega^{4} k_{i}^{2}\left[\left(1-\frac{\hbar \omega}{E_{k_{i}}}\right)-2\left(1-\frac{\hbar \omega}{E_{k_{i}}}\right)^{\frac{1}{2}} \cos \theta+1\right]}
$$

$\frac{d \sigma^{B 1}}{d \Omega}$

$=\frac{e^{6}}{\hbar^{2}(2 \pi)^{6}}\left(1-\frac{\hbar \omega}{E_{k_{i}}}\right)^{\frac{1}{2}} \frac{E_{o}{ }^{2}}{\omega^{4} 2 m E_{k_{i}}\left[\left(1-\frac{\hbar \omega}{E_{k_{i}}}\right)-2\left(1-\frac{\hbar \omega}{E_{k_{i}}}\right)^{\frac{1}{2}} \cos \theta+1\right]}$

$$
\begin{aligned}
& \frac{d \sigma^{B 1}}{d \Omega} \\
& =\frac{e^{6}}{(2)^{8} m \hbar^{2}(\pi)^{10} c^{5}}\left(1-\frac{l \hbar \omega}{E_{k_{i}}}\right)^{\frac{1}{2}} \frac{\lambda^{4} I_{o}}{E_{k_{i}}\left[\left(1-\frac{l \hbar \omega}{E_{k_{i}}}\right)-2\left(1-\frac{l \hbar \omega}{E_{k_{i}}}\right)^{\frac{1}{2}} \cos \theta+1\right]}
\end{aligned}
$$

We have the relation

$$
\omega=\frac{2 \pi c}{\lambda} \text { and } E_{o}^{2}=\frac{8 \pi I_{o}}{c}
$$

where,

$I_{o}$ is the intensity of the laser field.

$\lambda$ is the wave length of the radiation.

$c$ is the velocity of light.

$$
\frac{d \sigma^{B 1}}{d \Omega}=C\left(1-\frac{l \hbar \omega}{E_{k_{i}}}\right)^{\frac{1}{2}} \frac{\lambda^{4} I_{o}}{E_{k_{i}}\left[\left(1-\frac{l \hbar \omega}{E_{k_{i}}}\right)-2\left(1-\frac{l \hbar \omega}{E_{k_{i}}}\right)^{\frac{1}{2}} \cos \theta+1\right]}
$$

Where, $C=\frac{e^{6}}{(2)^{8} m \hbar^{2}(\pi)^{10} c^{5}}$ is the constant quantity.

Now, for $l=1$ i.e, one photon emission (stimulated emissioin)

$$
\frac{d \sigma^{B 1}}{d \Omega}=C\left(1-\frac{\hbar \omega}{E_{k_{i}}}\right)^{\frac{1}{2}} \frac{\lambda^{4} I_{O}}{E_{k_{i}}\left[\left(1-\frac{\hbar \omega}{E_{k_{i}}}\right)-2\left(1-\frac{\hbar \omega}{E_{k_{i}}}\right)^{\frac{1}{2}} \cos \theta+1\right]}
$$

This is the differential cross section for the scattering of an electron with one photon emission (stimulated Bremsstrahlung) in the presence of potential .

Also for $l=-1$ i.e, one photon absorption (inverse Bremsstrahlung),

$$
\frac{d \sigma^{B 1}}{d \Omega}=C\left(1+\frac{\hbar \omega}{E_{k_{i}}}\right)^{\frac{1}{2}} \frac{\lambda^{4} I_{o}}{E_{k_{i}}\left[\left(1+\frac{\hbar \omega}{E_{k_{i}}}\right)-2\left(1+\frac{\hbar \omega}{E_{k_{i}}}\right)^{\frac{1}{2}} \cos \theta+1\right]}
$$

This is the differential cross section for the scattering of an electron with one photon absorption (inverse Bremsstrahlung) in the presence of potential.

\section{Result and Discussion}

In the present work, we have studied the elastic scattering of an electron-atom interaction by absorbing a photon from the laser field. For simplicity, we have neglected the dressing effect of an atom i.e., we have neglected the field- atom interaction so that we can neglect the internal structure of an atom because we choose the intensity of the field in such a way that the target atom is transparent for the field.

Here, the differential cross-section equations (8) and (9) is found to be proportional to $\lambda^{4}$ (where $\lambda$ is the wavelength of the radiation ) which explains why it has been easier to observe laser -assisted cross section using infrared laser (high wave length and low frequency) than those operating in the visible or ultraviolet spectral regions. 
Equation (9) is plotted as the functions of scattering angle, kinetic energy of an electron, wavelength and intensity of the laser field as shown below:

1: Variation of differential scattering cross section with kinetic energy of the incident electron

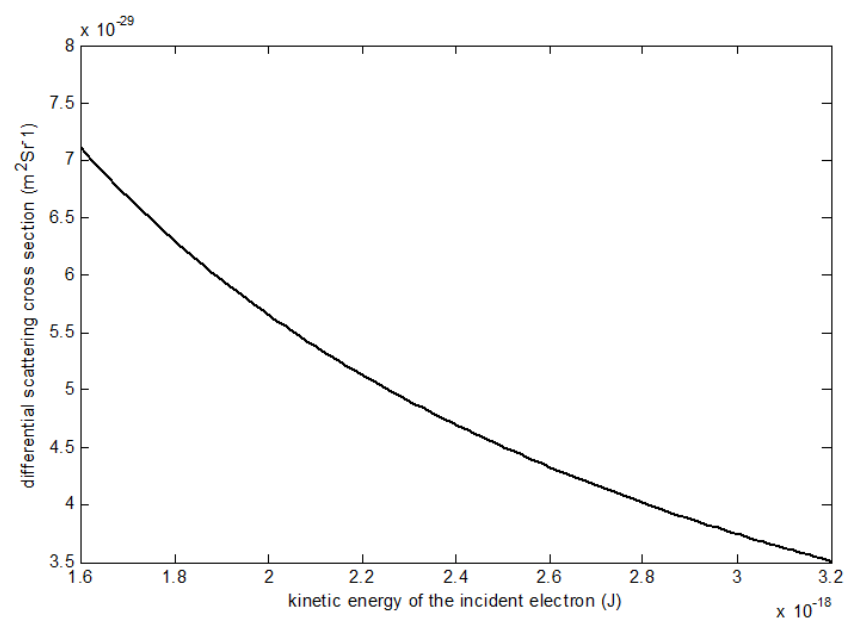

Fig. 1. Variation of differential scattering cross section with kinetic energy of the incident electron

From the plot, we see that the differential scattering cross section decrease as the kinetic energy of the incident electron increases. Going on increasing the kinetic energy of an incident electron we will get zero value of scattering cross section.

2: Variation of differential cross section with the wave length of the laser field

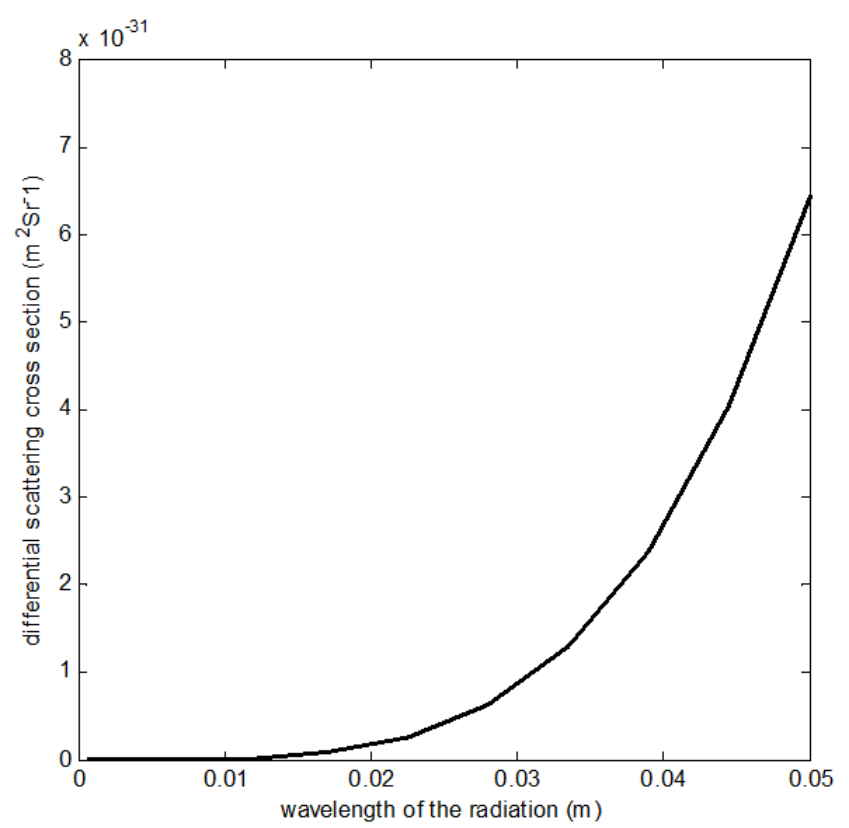

Fig. 2. Variation of differential cross section with wavelength of the field

From the plot, it is found that the differential scattering cross section increases with increase in wavelength of the laser field. It shows that the differential scattering cross section is zero below the wavelength $0.01 \mathrm{~m}$.
3: Variation of differential cross section with intensity of laser field

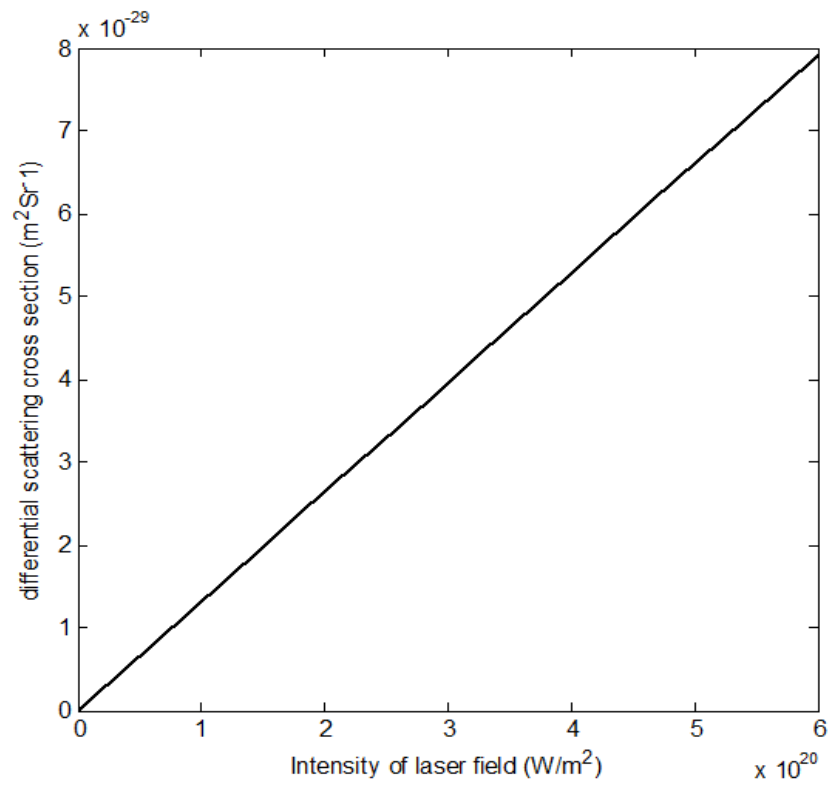

Fig. 3. Variation of differential cross section with intensity of the laser field

From the figure, it is clear that differential scattering cross section increase with increase in the intensity of the laser field. It shows that the differential scattering cross section varies linearly with intensity.

4: Variation of differential cross section with scattering angle

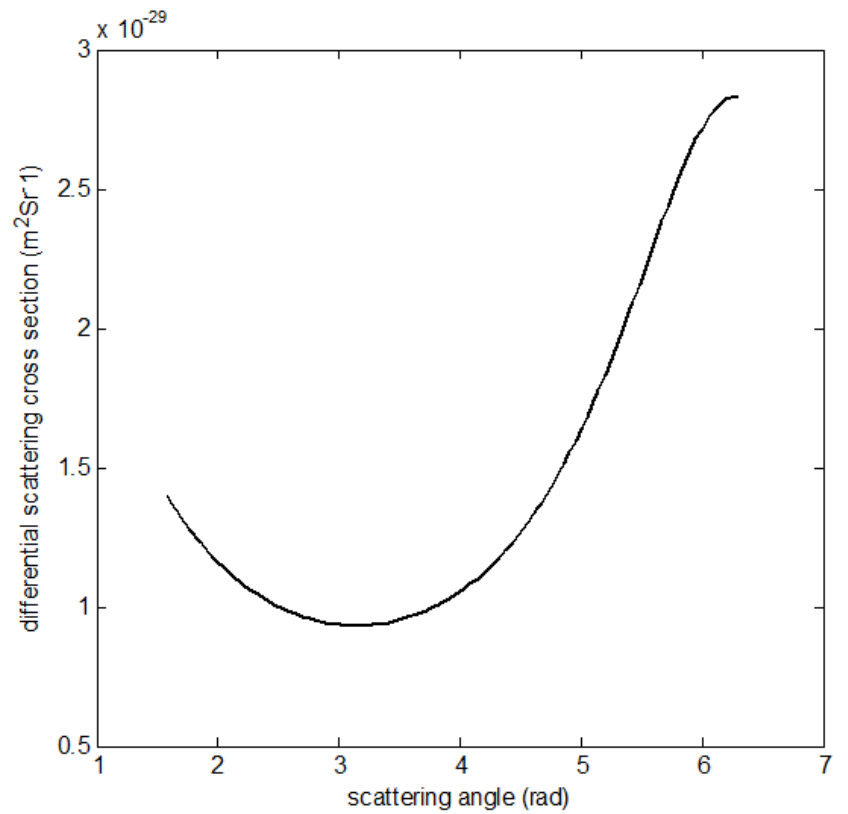

Fig. 4. Variation of differential cross section with scattering angle

From the plot, it is clear that differential cross section decreases as the scattering angle increase and attains the minimum value $1 \times 10^{-29} \mathrm{~m}^{2}$ and further increase in scattering angle also increase in differential scattering cross section which is clearly shown in polar plot. 
5: Polar plot of differential cross section with scattering angle

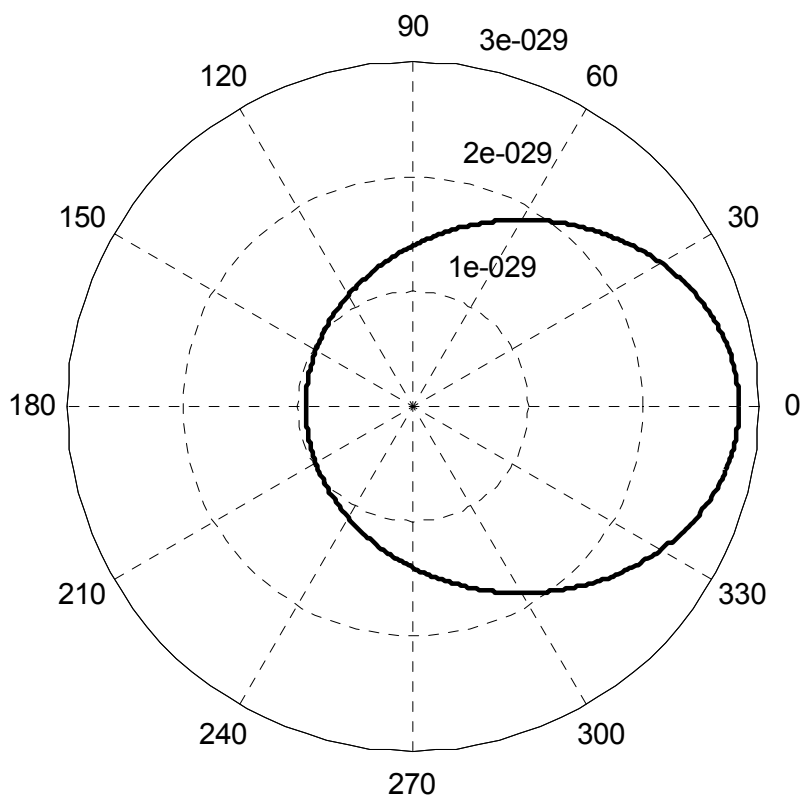

Fig. 5. Polar plot of differential cross section with scattering angle

From the polar plot, it is clear that the differential scattering cross section of an electron decreases with increase in scattering angle and attains minimum value and further increase in scattering angle also increase in cross section and attains maximum value $3 \times 10^{-29} \mathrm{~m}^{2}$.

It is generally observed that when electrons are scattered from the atom in the presence of a laser field, a new effect is observed which are not accessible in ordinary electron -atom scattering. This collision have the basic peculiarity of being processes in which three subsystem are present (i) the electron (ii) the target atom (iii) the radiation field. The last one provided energy and momentum and is characterized by the polarization of its electric field, which introduces in this collision process a new physical axis.

In this work, we have studied the scattering of an electron from the target atom by absorption of a photon from the laser field. From this study we concluded that the differential scattering cross section of an electron depends upon the intensity and wavelength of the laser field where as in ordinary electron -atom scattering, cross section only depends upon the scattering angle. The differential scattering cross section increases with increase in wavelength and intensity of the laser field. For a fixed value of a laser parameters and kinetic energy of an incident electron, the differential scattering cross section of an scattered electron decreases with increase in scattering angle and attains a minimum value of $1 \times 10^{-29} \mathrm{~m}^{2}=0.1 \mathrm{barn}$ and further increase in scattering angle also increase in differential scattering cross section and attains a maximum value of $3 \times 10^{-29} \mathrm{~m}^{2}=0.3 \mathrm{barn}$. Also from this study we see that, the differential scattering cross section for the electric field perpendicular to the direction of momentum transfer is zero. So from this study we concluded that the differential scattering cross section is also greatly depends upon the polarization of the laser field.

\section{For Polarized Potential}

If we choose $V(r)$ as polarized potential i. $e, V(r)=-\frac{\alpha_{p}}{2\left(r^{2}+d^{2}\right)^{2}}$

Where,

$$
d^{4}=\frac{\alpha_{p}}{2 z^{\frac{1}{3}}}
$$

$\alpha_{p} \rightarrow$ dipole polarizability

$\mathrm{Z} \rightarrow$ atomic number

Now,

Fourier Transformation of polarized potential,

$$
\begin{gathered}
\widehat{V}(\Delta)=-\frac{1}{2 \pi^{2}} \int_{0}^{\infty} \frac{\sin (\Delta r)}{\Delta} V(r) d r \\
\widehat{V}(\Delta)=\frac{1}{2 \pi^{2}} \int_{0}^{\infty} \frac{\sin (\Delta r)}{\Delta} \frac{\alpha_{p}}{2\left(r^{2}+d^{2}\right)^{2}} d r \\
=\frac{\alpha_{p}}{8 \pi^{2} i \Delta} \frac{\pi i \Delta}{2 d} e^{(-\Delta d)}[20] \\
\widehat{V}(\Delta)=\frac{\alpha_{p}}{16 \pi d} e^{(-\Delta d)}
\end{gathered}
$$

So that,

$$
\begin{aligned}
|\widehat{V}(\Delta)|^{2} & =\left|\frac{\alpha_{p}}{16 \pi d} e^{(-\Delta d)}\right|^{2} \\
& =\frac{\alpha_{p}{ }^{2}}{256 \pi^{2} d^{2}} e^{(-2 \Delta d)} \\
& =\frac{\alpha_{p}^{2}}{256 \pi^{2} d^{2}}\left[1-\frac{2 \Delta d}{1 !}+\frac{(2 \Delta d)^{2}}{2 !}-\cdots\right] \\
& |\widehat{V}(\Delta)|^{2}=\frac{\alpha_{p}^{2}}{256 \pi^{2} d^{2}}\left[1-2 \Delta d+\frac{(2 \Delta d)^{2}}{2 !}\right]
\end{aligned}
$$

Higher order terms can be neglected for small momentum transfer

$$
|\widehat{V}(\Delta)|^{2}=\frac{\alpha_{p}^{2}}{256 \pi^{2} d^{2}}-\frac{2 \alpha_{p}^{2} \Delta}{256 \pi^{2} d}+\frac{\alpha_{p}^{2}}{256 \pi^{2} d^{2}} \frac{(2 \Delta d)^{2}}{2}
$$

To calculate $\sum_{l} J_{l}\left(\Delta . \alpha_{o}\right)$ we have the expression for the Bessel function which is, [15]

$$
J_{n}(x)=\frac{x^{n}}{2^{n} \Gamma(\mathrm{n}+1)}\left[1-\frac{x^{2}}{2.2(n+1)}+\frac{x^{4}}{2.4 .2(n+1)(n+2)}-\ldots . .\right]
$$

Here we take

$$
\begin{gathered}
n=l \text { and } x=\Delta . \alpha_{o} \text { then } \\
J_{l}(x)=\frac{\left(\Delta . \alpha_{o}\right)^{l}}{2^{l} \Gamma(1+1)}\left[1-\frac{\left(\Delta . \alpha_{o}\right)^{2}}{2.2(l+1)}+\frac{\left(\Delta . \alpha_{o}\right)^{4}}{2.4 .2(l+1)(l+2)}-\ldots . .\right]
\end{gathered}
$$

for $l=1$ i.e, for one photon emission (stimulated Bremsstrahlung) then we get

$$
J_{l}\left(\Delta . \alpha_{o}\right)=\frac{\left(\Delta . \alpha_{o}\right)}{2 \Gamma(2)}\left[1-\frac{\left(\Delta . \alpha_{o}\right)^{2}}{2.2 .2}+\frac{\left(\Delta . \alpha_{o}\right)^{4}}{2.4 .2 .2 .3}-\ldots .\right]
$$




$$
J_{l}\left(\Delta . \alpha_{o}\right)=\frac{\left(\Delta \cdot \alpha_{o}\right)}{2}-\frac{\left(\Delta \cdot \alpha_{o}\right)^{2}}{2 \cdot 2 \cdot 2 \cdot 2}+\frac{\left(\Delta \cdot \alpha_{o}\right)^{4}}{2 \cdot 4 \cdot 2 \cdot 2 \cdot 3.2}-\ldots .
$$

If we take $\xi$ as the angle between electric field and momentum transfer i.e,

$$
\Delta \cdot \alpha_{o}=\Delta \alpha_{o} \cos \xi
$$

and for high frequency and low intensity the higher order terms of equation (4.97) can be neglected because $\alpha_{o}=\frac{e E_{o}}{\mathrm{~m} \omega^{2}}$.

Then we get,

$$
J_{l}\left(\Delta \cdot \alpha_{o}\right)=\frac{\Delta \alpha_{o} \cos \xi}{2}
$$

If the direction between electric field and momentum transfer is parallel (i.e, $\xi=0)$ then $\cos \xi=\cos 0=1$

Thus , $J_{l}\left(\Delta . \alpha_{o}\right)=\frac{\Delta \alpha_{o}}{2}$ and also,

$$
\left[J_{l}\left(\Delta \cdot \alpha_{o}\right)\right]^{2}=\frac{\Delta^{2} \alpha_{o}^{2}}{4}
$$

If the direction between electric field and momentum transfer is perpendicular (i.e, $\xi=90^{\circ}$ ) then $\cos \xi=$ $\cos 90^{\circ}=0$

Thus, $J_{l}\left(\Delta . \alpha_{o}\right)=0$.

for $l=-1$ one photon absorption (inverse Bremsstrahlung) the Bessel function is $J_{-1}\left(\Delta . \alpha_{o}\right)$.

We have the relation,

$$
J_{-l}(x)=(-1)^{l} J_{l}(x)
$$

Using this relation, for $l=1$ we get,

$$
\begin{aligned}
& J_{-1}\left(\Delta \cdot \alpha_{o}\right)=-J(x) \\
& J_{-1}\left(\Delta \cdot \alpha_{o}\right)=-\frac{\Delta \alpha_{o}}{2} \\
& {\left[J_{-1}\left(\Delta \cdot \alpha_{o}\right)\right]^{2}=\frac{\Delta^{2} \alpha_{o}{ }^{2}}{4}}
\end{aligned}
$$

We have,

$$
\begin{aligned}
\left|T_{k_{f} k_{i}}^{l}\right|^{2} & =\sum_{l} J_{l}^{2}\left(\Delta . \alpha_{o}\right)|\widehat{V}(\Delta)|^{2} \\
& =\frac{\Delta^{2} \alpha_{o}^{2}}{4}\left(\frac{\alpha_{p}^{2}}{256 \pi^{2} d^{2}}-\frac{2 \alpha_{p}^{2} \Delta}{256 \pi^{2} d}+\frac{\alpha_{p}^{2}}{256 \pi^{2} d^{2}} \frac{(2 \Delta d)^{2}}{2}\right) \\
& =\left(\frac{\Delta^{2} \alpha_{o}^{2} \alpha_{p}^{2}}{1024 \pi^{2} d^{2}}-\frac{2 \alpha_{o}^{2} \alpha_{p}{ }^{2} \Delta^{3}}{1024 \pi^{2} d}+\frac{\alpha_{o}{ }^{2}}{4} \frac{\alpha_{p}^{2}}{256 \pi^{2} d^{2}} \frac{(2 d)^{2} \Delta^{4}}{2}\right)
\end{aligned}
$$

Here the higher order term of momentum transfer is also neglected.

So that we get,

$$
\left|T_{k_{f} k_{i}}^{l}\right|^{2}=\frac{\alpha_{o}^{2} \alpha_{p}^{2}}{1024 \pi^{2} d^{2}} \Delta^{2}
$$

Thus we get,

$$
T_{k_{f} k_{i}}^{l}=\frac{\alpha_{o} \alpha_{p}}{32 \pi d} \Delta
$$

$$
\begin{gathered}
T_{k_{f} k_{i}}^{l}=\frac{\alpha_{p e E_{o}{ }^{2}}}{32 \pi m \omega^{2} d} \Delta \\
\alpha_{o}=\frac{e E_{o}}{m \omega^{2}}
\end{gathered}
$$

Now,

$$
\begin{gathered}
\frac{d \sigma}{d \Omega}=\frac{m^{2}}{(2 \pi)^{2} \hbar^{4}} \frac{k_{f}}{k_{i}}\left|T_{k_{f} k_{i}}^{l}\right|^{2} \\
\frac{d \sigma}{d \Omega}=\frac{m^{2}}{(2 \pi)^{2} \hbar^{4}}\left(1-\frac{l \hbar \omega}{E_{k_{i}}}\right)^{\frac{1}{2}} \frac{\alpha_{o}^{2} \alpha_{p}^{2}}{1024 \pi^{2} d^{2}} \Delta^{2} \\
\frac{d \sigma}{d \Omega}=\frac{m^{2} \alpha_{p}^{2}}{4096 \pi^{4} \hbar^{4} d^{2}}\left(1-\frac{l \hbar \omega}{E_{k_{i}}}\right)^{\frac{1}{2}} \alpha_{o}{ }^{2} \Delta^{2}
\end{gathered}
$$

Here,

$$
\begin{aligned}
\Delta & =k_{f}-k_{i} \text { thus } \\
\Delta^{2} & =\left(k_{f}-k_{i}\right)^{2} \\
& =k_{f}^{2}+k_{i}^{2}-2 k_{f} k_{i} \cos \theta
\end{aligned}
$$

Where, $\theta$ is the angle between $k_{f}$ and $k_{i}$, that means $\theta$ is the scattering angle between initial and final momentum of the projectile particle (electron).

$$
\left(\frac{\Delta}{k_{i}}\right)^{2}=\left(\frac{k_{f}}{k_{i}}\right)^{2}+1-2\left(\frac{k_{f}}{k_{i}}\right) \cos \theta
$$

$$
\begin{aligned}
\left(\frac{\Delta}{k_{i}}\right)^{2} & =\left(1-\frac{l \hbar \omega}{E_{k_{i}}}\right)-2\left(1-\frac{l \hbar \omega}{E_{k_{i}}}\right)^{\frac{1}{2}} \cos \theta+1 \\
\Delta^{2} & =k_{i}^{2}\left[\left(1-\frac{l \hbar \omega}{E_{k_{i}}}\right)-2\left(1-\frac{l \hbar \omega}{E_{k_{i}}}\right)^{\frac{1}{2}} \cos \theta+1\right]
\end{aligned}
$$

Substitute this value in equation (4.100) we get,

$$
\begin{gathered}
\frac{d \sigma}{d \Omega}=\frac{m^{2} \alpha_{p}^{2}}{4096 \pi^{4} \hbar^{4} d^{2}}\left(1-\frac{l \hbar \omega}{E_{k_{i}}}\right)^{\frac{1}{2}}\left(\frac{e E_{o}}{m \omega^{2}}\right)^{2} k_{i}^{2}\left[\left(1-\frac{l \hbar \omega}{E_{k_{i}}}\right)-\right. \\
21-l \hbar \omega E k i 12 \cos \theta+1
\end{gathered}
$$

we have from equation (4.83),

$$
\begin{gathered}
\frac{k_{f}}{k_{i}}=\left(1-\frac{l \hbar \omega}{E_{k_{i}}}\right)^{\frac{1}{2}} \\
\text { for } l=1 \text { it is } \\
\frac{k_{f}}{k_{i}}=\left(1-\frac{\hbar \omega}{E_{k_{i}}}\right)^{\frac{1}{2}}
\end{gathered}
$$

for $l=1$ i.e, for one photon emission (stimulated Bremsstrahlung) 


$$
\Delta^{2}=k_{i}^{2}\left[\left(1-\frac{\hbar \omega}{E_{k_{i}}}\right)-2\left(1-\frac{\hbar \omega}{E_{k_{i}}}\right)^{\frac{1}{2}} \cos \theta+1\right]
$$

Substitute this value in equation (4.102) we get,

$$
\begin{gathered}
\frac{d \sigma}{d \Omega}=\frac{m^{2} \alpha_{p}{ }^{2}}{4096 \pi^{4} \hbar^{4} d^{2}}\left(1-\frac{\hbar \omega}{E_{k_{i}}}\right)^{\frac{1}{2}}\left(\frac{e E_{o}}{m \omega^{2}}\right)^{2} k_{i}^{2}\left[\left(1-\frac{\hbar \omega}{E_{k_{i}}}\right)\right. \\
\left.-2\left(1-\frac{\hbar \omega}{E_{k_{i}}}\right)^{\frac{1}{2}} \cos \theta+1\right] \\
\frac{d \sigma}{d \Omega}=\frac{m^{2} \alpha_{p}^{2}}{4096 \pi^{4} \hbar^{6} d^{2}}\left(1-\frac{\hbar \omega}{E_{k_{i}}}\right)^{\frac{1}{2}} \frac{e^{2} E_{o}{ }^{2}}{m^{2} \omega^{4}} \hbar^{2} k_{i}^{2}\left[\left(1-\frac{\hbar \omega}{E_{k_{i}}}\right)-\right. \\
21-\hbar \omega E k i 12 \cos \theta+1
\end{gathered}
$$

$$
\begin{gathered}
\frac{d \sigma}{d \Omega}=\frac{m e^{2} \alpha_{p}{ }^{2}}{32768 \pi^{8} \hbar^{6} d^{2} c^{4}}\left(1-\frac{\hbar \omega}{E_{k_{i}}}\right)^{\frac{1}{2}} E_{o}{ }^{2} E_{k_{i}} \lambda^{4}\left[\left(1-\frac{\hbar \omega}{E_{k_{i}}}\right)\right. \\
\left.-2\left(1-\frac{\hbar \omega}{E_{k_{i}}}\right)^{\frac{1}{2}} \cos \theta+1\right] \\
\frac{d \sigma}{d \Omega}=C\left(1-\frac{\hbar \omega}{E_{k_{i}}}\right)^{\frac{1}{2}} E_{o}{ }^{2} E_{k_{i}} \lambda^{4}\left[\left(1-\frac{\hbar \omega}{E_{k_{i}}}\right)-2\left(1-\frac{\hbar \omega}{E_{k_{i}}}\right)^{\frac{1}{2}} \cos \theta+1\right]
\end{gathered}
$$

Where, $\mathrm{C}=\frac{m e^{2} \alpha_{p}{ }^{2}}{32768 \pi^{8} \hbar^{6} d^{2} c^{4}}$ (constant)

This is the differential cross section for the scattering of an electron with one photon emission (stimulated Bremsstrahlung) in the presence of potential V(r).

Again from equation (14) we have,

$$
\frac{k_{f}}{k_{i}}=\left(1-\frac{l \hbar \omega}{E_{k_{i}}}\right)^{\frac{1}{2}}
$$

for $l=-1$,one photon absorption (inverse Bremsstrahlung) it is

$$
\frac{k_{f}}{k_{i}}=\left(1+\frac{\hbar \omega}{E_{k_{i}}}\right)^{\frac{1}{2}}
$$

Also, $\Delta^{2}=k_{i}^{2}\left[\left(1+\frac{\hbar \omega}{E_{k_{i}}}\right)-2\left(1+\frac{\hbar \omega}{E_{k_{i}}}\right)^{\frac{1}{2}} \cos \theta+1\right]$

Then equation (4.102) becomes

$$
\begin{gathered}
\frac{d \sigma}{d \Omega}=\frac{m^{2} \alpha_{p}^{2}}{4096 \pi^{4} \hbar^{6} d^{2}}\left(1+\frac{\hbar \omega}{E_{k_{i}}}\right)^{\frac{1}{2}} \frac{e^{2} E_{o}{ }^{2}}{m^{2} \omega^{4}} \hbar^{2} k_{i}^{2}\left[\left(1+\frac{\hbar \omega}{E_{k_{i}}}\right)-\right. \\
21+\hbar \omega E k i 12 \cos \theta+1
\end{gathered}
$$

This is the differential cross section for the scattering of an electron with one photon absorption (inverse Bremsstrahlung) in the presence of potential $\mathrm{V}(\mathrm{r})$.

Where,

$m=$ mass of an elactron

$k_{i}=$ initial momentum vector of an electron

$E_{k_{i}}=$ initial kinetic energy of the incident an electron

$\hbar \omega=$ photon energy of the laser

$l=$ no. of the photon transfer during interaction

$\theta=$ scattering angle

$\Delta=$ momentum transfer

$E_{o}=$ amplitude of the electric field of the laser

\section{Result and Discussion}

\subsection{Result and Discussion}

In the present thesis work, we have studied the elastic scattering of an electron-atom interaction by absorbing a photon from the laser field. For simplicity, we have neglected the dressing effect of an atom [21] i.e., we have neglected the field- atom interaction so that we can neglect the internal structure of an atom because we choose the intensity of the field in such a way that the target atom is transparent for the field.

When an electron scatters elastically from the target by absorbing a photon (inverse Bremsstrahlung, i.e., $l=-1$ ) from the fields, then the differential scattering cross-section is given by equation (17) as,

$$
\begin{gathered}
\frac{d \sigma}{d \Omega}=\frac{m^{2} \alpha_{p}^{2}}{4096 \pi^{4} \hbar^{6} d^{2}}\left(1+\frac{\hbar \omega}{E_{k_{i}}}\right)^{\frac{1}{2}} \frac{e^{2} E_{o}{ }^{2}}{m^{2} \omega^{4}} \hbar^{2} k_{i}^{2}\left[\left(1+\frac{\hbar \omega}{E_{k_{i}}}\right)\right. \\
\left.-2\left(1+\frac{\hbar \omega}{E_{k_{i}}}\right)^{\frac{1}{2}} \cos \theta+1\right]
\end{gathered}
$$

From this relation, it is clear that the frequency $\omega$ of the laser field is not only important but also the field amplitude $E_{o}$ is important for the differential cross section.

Now we changed this equation in terms of wavelength and the intensity of the laser field.

We have the relations,

$$
E_{o}^{2}=\frac{8 \pi I_{o}}{c}
$$

where, $I_{o}$ is the intensity of the laser field.

and also, $\omega=\frac{2 \pi c}{\lambda}$

where

$\lambda$ is the wave length of the radiation.

$c$ is the velocity of light.

Substituting the values of $E_{o}^{2}$ and $\omega$ in equation (17),then we get the following form for the differential cross- section,

$$
\frac{d \sigma}{d \Omega}=\frac{2 m e^{2} \alpha_{p}{ }^{2}}{4096 \pi^{4} \hbar^{6} d^{2}}\left(1+\frac{\hbar \omega}{E_{k_{i}}}\right)^{\frac{1}{2}} \frac{\left(\frac{8 \pi I_{o}}{c}\right) E_{k_{i}} \lambda^{4}}{(2 \pi c)^{4}}\left[\begin{array}{c}
\left(1+\frac{\hbar \omega}{E_{k_{i}}}\right)- \\
2\left(1+\frac{\hbar \omega}{E_{k_{i}}}\right)^{\frac{1}{2}} \cos \theta+1
\end{array}\right]
$$




$$
\begin{gathered}
\frac{d \sigma}{d \Omega}=\frac{m e^{2} \alpha_{p}^{2}}{32768 \pi^{8} \hbar^{6} d^{2} c^{4}}\left(1+\frac{\hbar \omega}{E_{k_{i}}}\right)^{\frac{1}{2}}\left(\frac{8 \pi I_{o}}{c}\right) E_{k_{i}} \lambda^{4}\left[\left(1+\frac{\hbar \omega}{E_{k_{i}}}\right)-\right. \\
21+\hbar \omega E k i 12 \cos \theta+1
\end{gathered}
$$

Similarly, when an electron scatters elastically from the target by emitting a photon (stimulated Bremsstrahlung, i.e., $l=1$ ), then the differential scattering cross-section is given by equation (18) as,

$$
\begin{gathered}
\frac{d \sigma}{d \Omega}=\frac{m^{2} \alpha_{p}^{2}}{4096 \pi^{4} \hbar^{6} d^{2}}\left(1-\frac{\hbar \omega}{E_{k_{i}}}\right)^{\frac{1}{2}} \frac{e^{2} E_{o}{ }^{2}}{m^{2} \omega^{4}} \hbar^{2} k_{i}^{2}\left[\left(1-\frac{\hbar \omega}{E_{k_{i}}}\right)-\right. \\
21-\hbar \omega E k i 12 \cos \theta+1
\end{gathered}
$$

Substituting the values of $E_{o}^{2}$ and $\omega$ in equation (5.3), then we get the following form for the differential cross- section,

$$
\begin{gathered}
\frac{d \sigma}{d \Omega}=\frac{m e^{2} \alpha_{p}^{2}}{32768 \pi^{8} \hbar^{6} d^{2} c^{4}}\left(1-\frac{\hbar \omega}{E_{k_{i}}}\right)^{\frac{1}{2}}\left(\frac{8 \pi I_{o}}{c}\right) E_{k_{i}} \lambda^{4}\left[\left(1-\frac{\hbar \omega}{E_{k_{i}}}\right)-\right. \\
21-\hbar \omega E k i 12 \cos \theta+1
\end{gathered}
$$

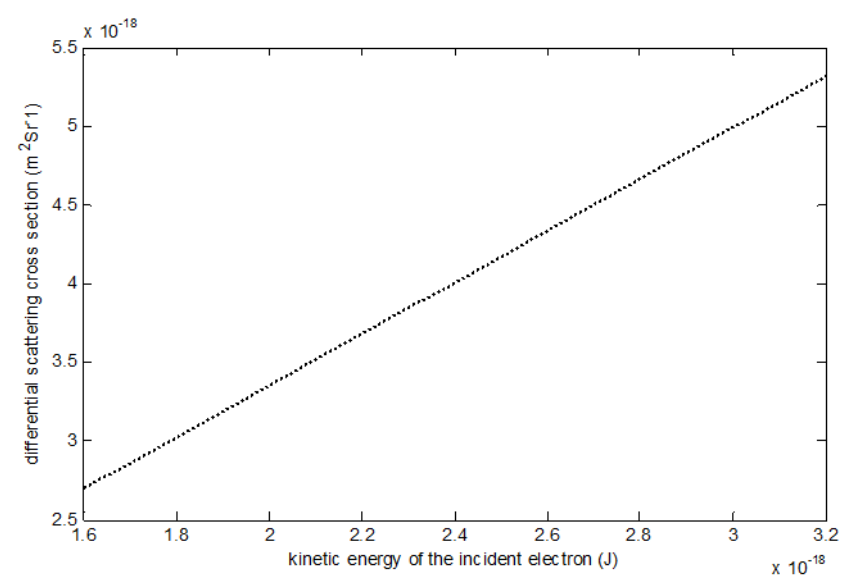

Fig 6. Variation of differential scattering cross section with kinetic energy of the incident electron

Here, the differential cross-section equations (18) and (19) is found to be proportional to $\lambda^{4}$ (where $\lambda$ is the wavelength of the radiation ) which explains why it has been easier to observe laser -assisted cross section using infrared laser (high wave length and low frequency) than those operating in the visible or ultraviolet spectral regions[22].

Equation (18) and (19) are plotted as the functions of scattering angle, kinetic energy of an electron, wavelength and intensity of the laser field as shown below:

From the plot, we see that the differential scattering cross section increases as the kinetic energy of the incident electron increases. Going on increasing the kinetic energy of an incident electron we will get infinite value of scattering cross section.

\subsection{Variation of Differential Cross Section with the Wave Length of the Laser Field}

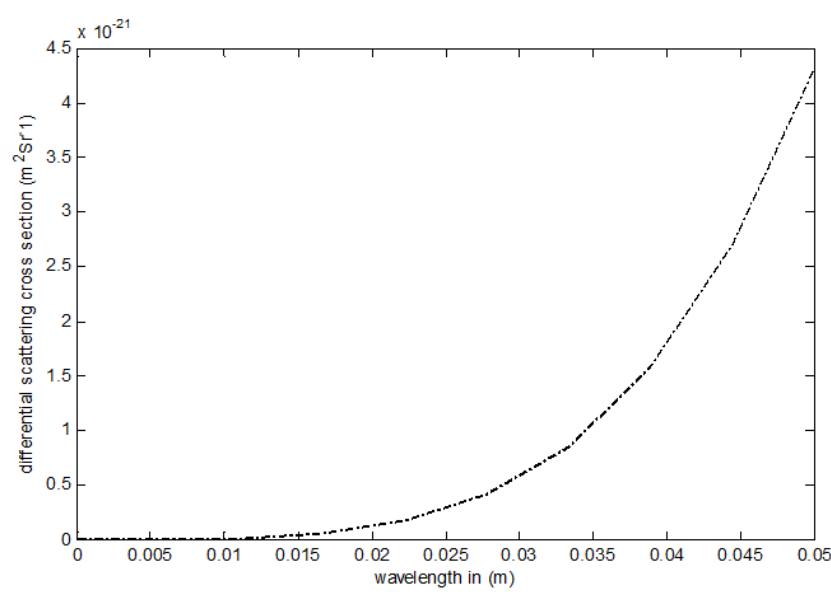

Fig 7. Variation of Differential Cross Section with the Wave Length of the Laser Field

From the plot, it is found that the differential scattering cross section increases with increase in wavelength of the laser field. It shows that the differential scattering cross section is zero below the wavelength $0.015 \mathrm{~m}$.

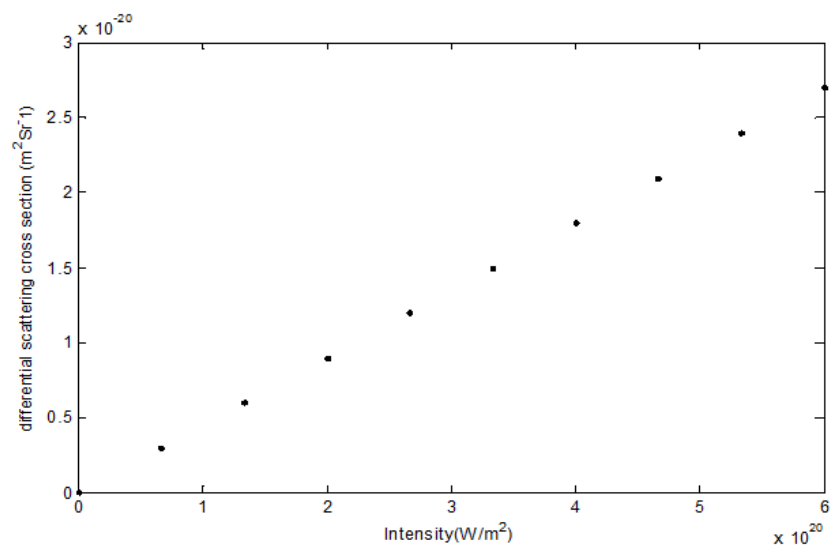

Fig 8. Variation of differential cross section with intensity of the laser field

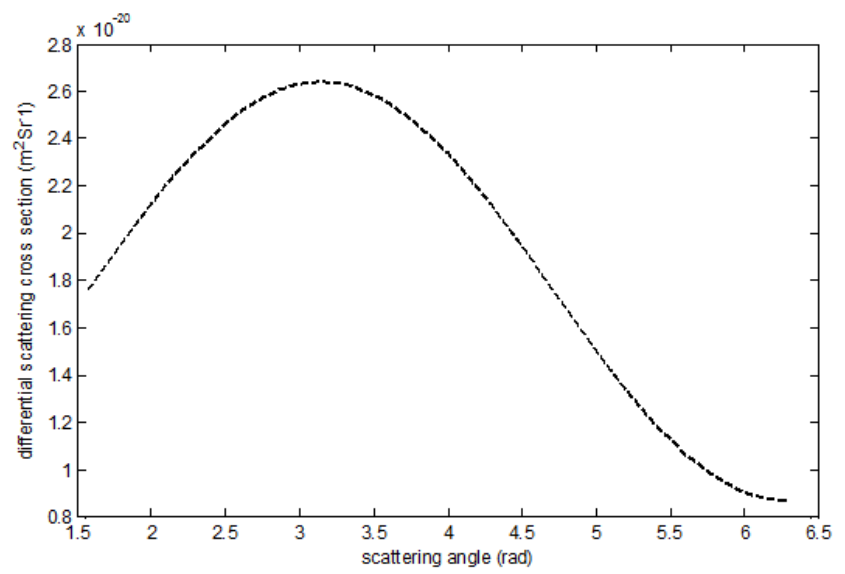

Fig 9. Variation of differential cross section with scattering angle

From the figure, it is clear that differential scattering cross section increase with increase in the intensity of the laser 
field. It shows that the differential scattering cross section varies linearly with intensity.

From the plot, it is clear that differential cross section increases as the scattering angle increase and attains the maximum value $2.6 \times 10^{-20} \mathrm{~m}^{2}$ and further increase in scattering angle decreases in differential scattering cross section which is clearly shown in polar plot[23].

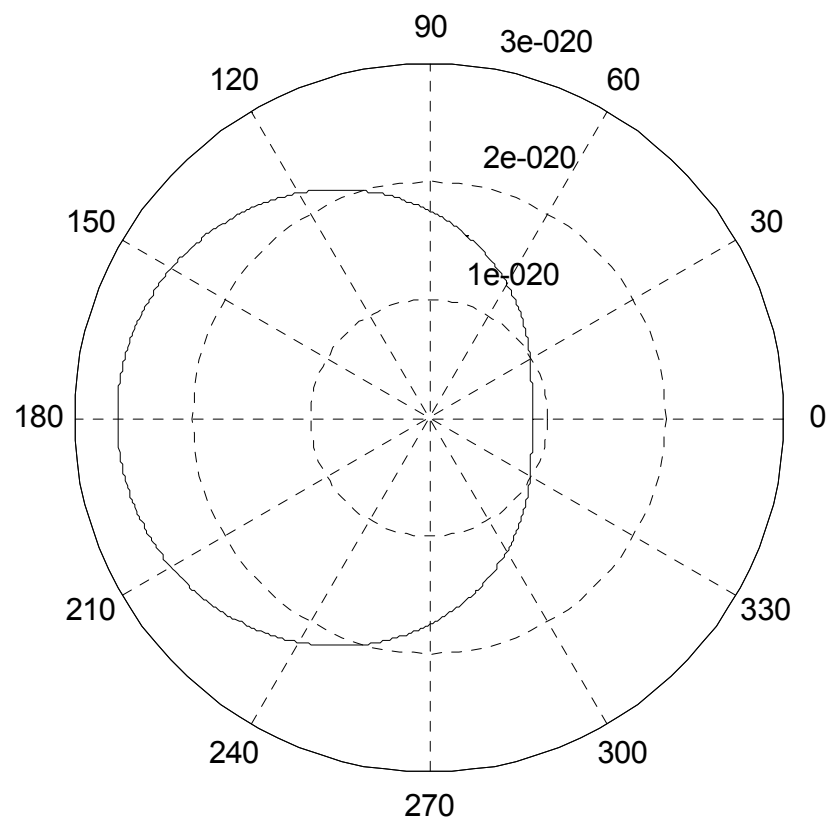

Fig 10. Polar plot of differential cross section with scattering angle

From the polar plot, it is clear that the differential scattering cross section of an electron increase with increase in scattering angle and attains maximun value of $2.6 \times$ $10^{-20} \mathrm{~m}^{2}$ and further increase in scattering angle, decreases in cross section and attains minimum value of $0.98 \times 10^{-20} \mathrm{~m}^{2}$.

\subsection{Conclusion}

It is concluded that from the above discussion that when electrons are scattered from the atom in the presence of a laser field, a new effect is observed which are not accessible in ordinary electron atom scattering. This collision have the basic peculiarity of being processes in which three subsystem are present (i) the electron (ii) the target atom (iii) the radiation field. The last one provided energy and momentum and is characterized by the polarization of its electric field, which introduces in this collision process a new physical axis.

This work reflected that the scattering of an electron from the target atom by absorption of a photon from the laser field. From this study we concluded that the differential scattering cross section of an electron depends upon the intensity and wavelength of the laser field where as in ordinary electron atom scattering, cross section only depends upon the scattering angle. The differential scattering cross section increases with increase in wavelength and intensity of the laser field. For a fixed value of a laser parameters and kinetic energy of an incident electron, the differential scattering cross section of an scattered electron increases with increase in scattering angle and attains a maximum value of $2.6 \times$ $10^{-20} \mathrm{~m}^{2}$ and further increase in scattering angle, decreases in differential scattering cross section and attains a minimum value of $0.98 \times 10^{-20} \mathrm{~m}^{2}$.

\section{References}

[1] J. Mathews and R.L. Walker, Mathematical Methods of Physics, Second Edition, California Institute of Technology, copyright (C) 1970 by Pearson Education (2005).

[2] C. Harper, Introduction to Mathematical physics, Department of Physics California State University, Hayward, Prentice Hall of India Pvt. Ltd, New Delhi-110 001 (2006)

[3] J. D. Jackson, Classical Electrodynamics, Third Edition, John Wiley and Sons, 709 (1971) .

[4] A.A Balakin and G.M.Frainman, Bremsstrahlung in a strong Laser field JEPT, 93, 695, (2001).

[5] G.M Frainman, V.A.Mironov and A.A Balakin , Representative Electrons and Energy Exchange in Strong Laser field, Phys.Rev.Lett, 82, 319 (1999).

[6] F.W. Byron, P.Francken and C.J. Joachain, Laser assisted elastic electron atom collision, J. Phys: B: At. Mol. Phys. 20 5487-5503 (1987).

[7] N.M Kroll and KM Waston, Phys. Rev. A 8, 804(1973).

[8] J.R. Reitz, F.J. Milford, R. W. Christy, Foundation of electromagnetic theory, Third Edition, Narosa Publishing House, New Delhi (1998).

[9] H. Goldstein, C. Poole, J. Safko, Classical Mechanics, third edition, Pearson Education, India (2007).

[10] F. Ehlotzky - Fundamentals of Laser Interaction -Springer (1985).

[11] Marvin H. Mittleman, Introduction to the Theory of Laser Atom Interaction, second edition, Printed in the United State of America (1993).

[12] A.G.Sitenko and P.J. Shepherd, Lectures in Scattering Theory.

[13] J.J. Sakurai- Modern Quantum Mechanics Revised Edition, Addition-Wesley Publishing Company (1994).

[14] B.H Bransden, W.A. Benjamin, New York - Atomic Collision Theory, standard book number 8053-1180-7 (C) (1970).

[15] G.N. Watson- Theory Of Bessel Function, Cambridge at the University Press 1922.

[16] M. Hannachi, Z. Rouabah, C. Champion, N. Bouarissam Journal of Electron Spectroscopy and Related Phenomena, Volume 195, August 2014, Pages 155-159

[17] A. Bekzhanov, S. Bondarenko, V.urovNuclear Physics B Proceedings Supplements, Volume 245, December 2013, Pages 65-68

[18] H. Aouchiche, F. Medegga, C. Champion Nuclear Instruments and Methods in Physics Research Section B: Beam Interactions with Materials and Atoms, Volume 333, 15 August 2014, Pages 113-119 
[19] E. Merzbacher-Quantum Mechanics, Third edition, John Wiley and Sons, Inc (1998).

[20] N. Zettili - Quantum Mechanics Concepts and Application, second edition, John Wiley and Sons, Ltd (2009).

[21] M. L. Goldberger- Collision Theory, John Wiley and Sons, Inc, Third Printing (1967).
[22] Laser-Assisted Elastic Electron scattering From Argon, Theor. Phys.(Beijing, China) 51 (2009) pp (131-134).

[23] S. Balasubramian, Oxford and IBH Publishing Co. New Delhi, Bombay, Calcutta, 1985. 